\title{
On the nature of discrete radio ejection events in microquasars
}

\section{Prat and J. Rodriguez}

DSM/IRFU/Service d'Astrophysique, CEA/Saclay, F-91191 Gif-sur-Yvette, France

E-mail: lionel.pratecea.fr

The onset of most microquasar outbursts is characterized by a state transition between a Low/Hard State (LHS) and a High/Soft State (HSS). Besides drastic spectral and timing changes, this transition often shows a discrete ejection event detectable in the radio range. However, the exact nature of the ejected material and the mechanisms that give birth to these phenomena are yet to be unraveled. Recent simultaneous radio and X-ray observations on several sources point to a coronal nature of the ejected material. First, in the case of XTE J1550-564, the hard X-ray tail, usually attributed to an inverse Compton effect, disappears just before an ejection is detected at radio wavelengths. Then, in the case of GRS 1915+105, the flux of the Compton component decreases sharply just prior to an ejection. We interpret this behaviour as evidence of the disappearance of the corona, which is ejected under the form of blobs. In the case of the 2002 outburst of GX 339-4, the first radio detection occurs after a decrease of the disc flux, which may indicate that the radio events are initiated by the disc. The peak of the radio flare occurs several day after, and in particular after a large decrease of the coronal flux. Finally, in the case of XTE J1859+226, drastic physical changes occurred in the corona just before the state transition, compatible with the disappearance of part of this medium. Thus, a total of 4 microquasars point in the direction of an ejection of the corona, feature that is yet to be confirmed (or infirmed) in the case of other available sources.

VII Microquasar Workshop: Microquasars and Beyond September 1-5 2008

Foca, Izmir, Turkey 


\section{Introduction}

Up to now, about 20 black-hole X-ray transients have been observed, along with $\sim 20$ more candidate black-hole binaries (McClintock et al. [8]). This led to the identification of several spectral states, depending both on the spectral and temporal characteristics of the source. The onset of a microquasar outburst usually occurs in a Low/Hard State (LHS, following the classification by Homan et al. [5]), dominated by a hard X-ray emission from a relativistic jet and/or from a so-called coronal component, combined with strong temporal variability and Quasi-Periodic Oscillations (QPOs). The object then evolves, through a state transition, into a High/Soft State (HSS) dominated by soft X-ray emission from the accretion disk and less temporal variability. Another state transition usually occurs at the end of the outburst, when the source goes back in the LHS. This simple general picture is often complicated by the presence of intermediate states, mixture of the canonical LHS and HSS: the Hard InterMediate State (HIMS) and the Soft InterMediate State (SIMS).

A key to fully understand the physics of these states may reside in deeper studies of the state transitions. These phenomena involve fast and drastic changes in the system, and show strong connexions between the relativistic jet and the accretion disk. The first transition, between the HIMS and the SIMS, often shows a discrete ejection event detectable in the radio range, simultaneous with a rapid increase of the disk flux and a quenching of the jet emission. Thus, the use of simultaneous radio and X-ray observations is necessary to monitor adequately the events happening during this transition. Such simultaneous monitoring is available for a handful of sources only, but their study may already lead to good constraints on the evolution of the coronal part of the system, as we show here.

\section{XTE J1550-564 and GRS 1915+105: probable ejection of the corona}

In 2000, the $\sim 70$ day outburst of XTE J1550-564 was observed thoroughly by the RXTE satellite. Corbel et al. [3] report the detection of radio emission, which they associated to the first state transition (Fig. 1), although the precise date of the emission could not be determined. In the $\mathrm{X}$-ray range, a power-law with an exponential cut-off adequately describes the spectra before the state transition. This feature is widely attributed to a thermal comptonization process, characteristic of the so-called coronal medium. The evolution of this component is clearly correlated with the state transitions: it disappears $\sim 2$ days after the first transition, on MJD 51662, and reappears after the second transition back to the LHS, on MJD 51682. In the days after MJD 51662, the diskblackbody flux remains approximately constant during an initial period, while the power-lax flux decreases significantly. Given the relative constancy of the photon index on those days, this may suggest that there is less Compton up-scattering of the soft photons. In other words, part of the Compton medium disappears at about the same time a radio ejection is detected. It is thus tempting to consider that the Compton medium may be blown away.

GRS $1915+105$ is a very peculiar source, which shows a wider and faster range of behaviour than the other microquasars. This source has also stayed in outburst at least for the last $\sim 15$ years, giving a rare luxury of data to understand accretion and ejection physics. In its case, a joint $I N$ TEGRAL and RXTE monitoring campaign follows the source since 2003. The RXTE observations 


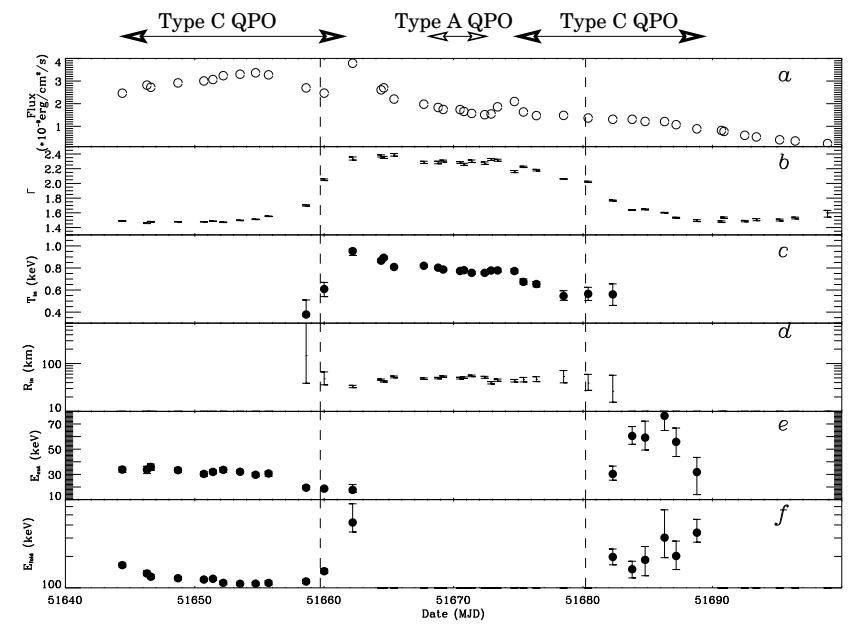

Figure 1: Evolution of the spectral parameters of XTE 1550-564 over its 2000 outburst. (a) 2-200 keV light curve. (b) Power-law spectral index $\Gamma$. (c) Inner disk temperature (keV). (d) Inner disk radius (assuming D $=6 \mathrm{kpc}$ and $\left.\mathrm{i}=70 \cdot 1^{\circ}\right) .(e)$ Cutoff energy $(\mathrm{keV}) .(f)$ The $e$-folding of the cutoff $(\mathrm{keV})$. The vertical dashed lines represent the dates of state transitions, the first transition being accompanied by a radio flare (from Rodriguez et al. [10]).

allowed the identification of 12 separate classes (Belloni et al. [1]), which could be interpreted as transitions between three basic spectral states: a Hard Intermediate one (State C) and two Soft Intermediate ones (State A and State B). Among the 12 classes, the $v, \theta, \alpha$ and $\beta$ classes show $\mathrm{X}$-ray cycles, corresponding to transitions between the $\mathrm{A}, \mathrm{B}$ and $\mathrm{C}$ states. In the radio range, they show a strong radio variability, interpreted as discrete ejections of matter.

Fig. 2 shows radio and X-ray lightcurves of GRS 1915+105 (two top panels). On these curves, X-ray dips (I) are followed by a spike (II) and, $\sim 0.3$ hour after the spike, by a radio ejection. In a broader study, including more spectral classes, we have recently pinpointed the strong similarities between every spectral classes characterized by X-ray cycles (including the $\lambda$ class), thus generalizing this behaviour for wider parts of this source's evolution (Rodriguez et al. [11]). In these
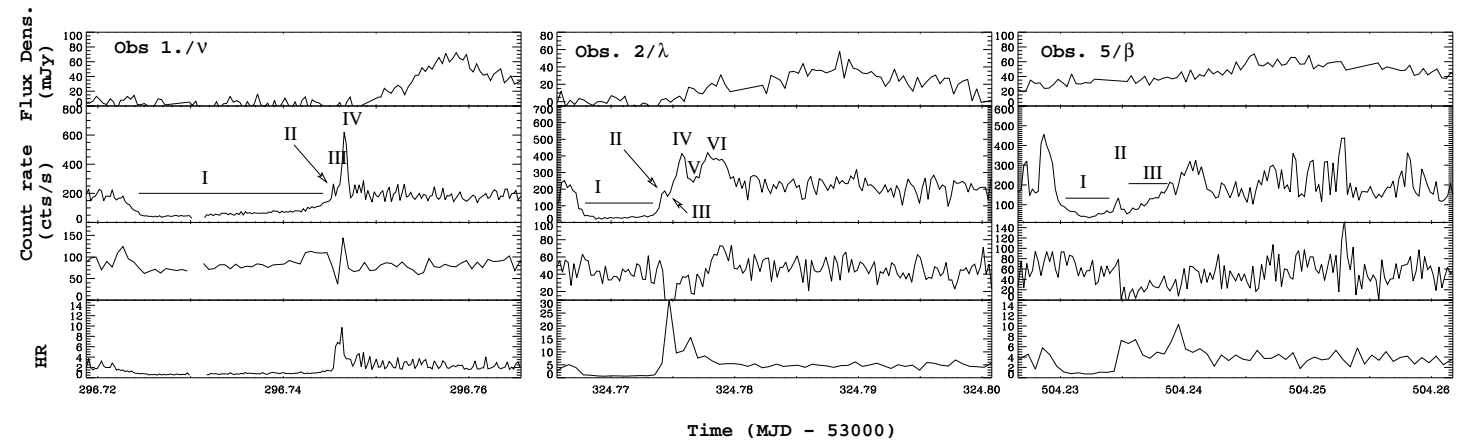

Figure 2: Three cycle lightcurves, corresponding to the $v, \lambda$ and $\beta$ spectral classes of GRS $1915+105$. From top to bottom, the panels represent respectively the Ryle $15 \mathrm{GHz}$, the INTEGRAL/JEM-X 3-13 keV, the INTEGRAL/ISGRI 18-50 keV lighcurves, and the 3-13 keV/18-50 keV Hardness Ratio (from Rodriguez et al. [11]). 

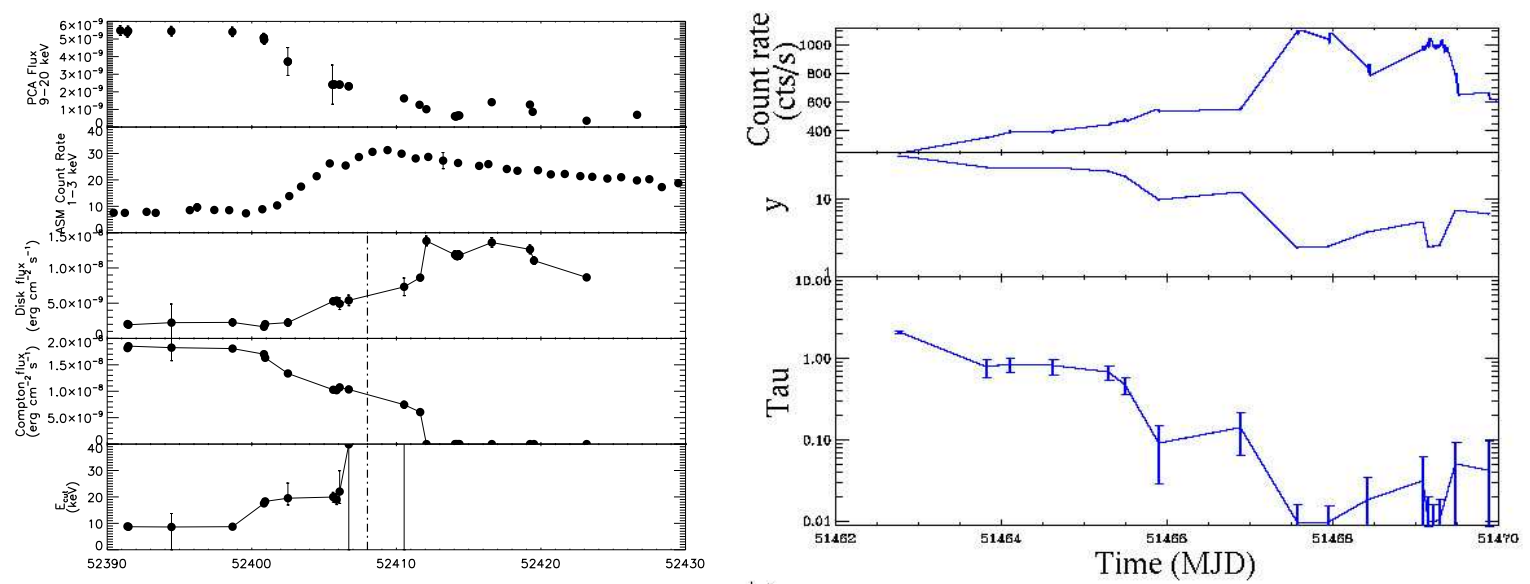

Figure 3: Left: Spectral evolution of GX 339-4 at the beginning of its 2002 outburst. From top to bottom, the panels represent respectively the RXTE/PCA 9-20 keV lightcurve, the RXTE/ASM 1-3 keV lightcurve, the disk flux, the Compton component flux and the high-energy cutoff value. The vertical dot-dashed line marks the detection of a radio ejection. The forth and fifth panels mark the coronal parameters: the coronal flux starts to fade $\sim 6$ days before the ejection, and fall below the detection level $\sim 2$ days before the ejection. Right: Spectral evolution of XTE J1859+226 over its 1999 outburst. From top to bottom, the panels represent respectively the $R X T E / P C A$ lightcurve, the Comptonization parameter $y=k T_{e} \max \left(\tau, \tau^{2}\right)$ and the optical depth $\tau$.

observations, we showed that each spike is indicative of the disappearance of the Compton component from the X-ray spectra. This again points to an ejection of (part of) the corona, which is then detected in the radio range.

\section{Two more candidates: GX 339-4 and XTE J1859+226}

\subsection{GX 339-4: disappearance of the corona suspected in two outbursts}

GX 339-4 is a well-known X-ray binary, which underwent at least 10 outbursts since its discovery in 1972 (Kong et al. [7]). The two outbursts that occurred in 1998 and 2002 were followed by simultaneous RXTE observations and radio observations. The strong anticorrelation between the radio and hard X-ray fluxes in 1998 have already led Fender et al. [4] to suspect a reduction of the coronal medium at the beginning of the HSS. At that time, only RXTE/ASM observations were available, which did not permit any precise spectral analysis of this state transition.

The 2002 outburst was followed by RXTE/PCA (e.g. Homan et al. [6]), from which some spectral results are reported on Fig. 3, left. A radio flare was detected on MJD 52408, which is indicated by a vertical line on the plot. The coronal flux (forth panel starting from the top) started to fade $\sim 6$ days before this outburst, until MJD 52412 when no cut-off is detected anymore. In other words, the Compton medium fainters drastically a few days before a strong ejection of matter is detected at radio wavelength: the corona may be gradually ejected just before the state transition.

\subsection{XTE J1859+226: major change in the coronal material}

The candidate back-hole binary XTE J1859+226 was observed by RXTE during its 1999 out- 
burst. On Fig. 3, right, we report from top to bottom the evolution of the RXTE/PCA count rate, the Comptonization parameter $y \propto k T_{e} \max \left(\tau, \tau^{2}\right)$ (Rybicki \& Lightman [9]), and the optical depth $\tau$. The parameters plotted here correspond to the period of a state transition between a HIMS and a SIMS, at the beginning of the outburst. $y$ is a measure of the efficiency of the Comptonization process, while $\tau$ is proportional to the product of the density of the medium $\rho$, and its typical size $\mathrm{R}: \tau \propto \rho R$. Thus, a diminution of $\tau$ either corresponds to a diminution of the density of the coronal medium, or to a diminution of its size. In both cases, this medium looses matter just prior to the state transition. Moreover, this evolution occurred at the time of a radio ejection, detected on MJD 51468 (Brocksopp et al. [2]). This shows that, again, in the case of XTE J1859+226, the state transition is characterized by a major change in the coronal medium, which may indicate that part of this medium is ejected by the system.

\section{Conclusions}

In the cases of the 4 sources studied here, a similar behaviour is detected. An increase of the soft $\mathrm{X}$-ray spectrum and a softening of the $\mathrm{X}$-ray flux leads to an ejection that occurs $\sim 1-3$ days after, detected in the radio range. Spectral fits show that prior to this ejection, the coronal medium parameters change drastically, until this component becomes undetectable. It is thus tempting to link the coronal changes to the ejection of matter. Note that a fifth source, XTE J1748-288, may also show a similar behaviour, and is still under study.

This interpretation still needs to be confirmed by a detailed analysis of the spectral behaviour of more back-hole binaries, in the vicinity of state transitions: the coronal emission lies in the highenergy end of the available X-ray spectra, which renders it difficult to detect and monitor. However, if this phenomenon is confirmed, it will put strong constraints on disk accretion models and, ultimately, provide a new insight on the interplay between the different components that surround an accreting compact object.

\section{References}

[1] T. Belloni, M. Klein-Wolt, M. Mendez, M. van der Klis, \& J. van Paradijs, 2000 A\&A, 355, 271

[2] C. Brocksopp, R. Fender, M. McCollough, G. Pooley, M. Rupen, et al., 2002 MNRAS, 331, 765

[3] S. Corbel, P. Kaaret, R. K. Jain, C. D. Bailyn, R. Fender, et al., 2001 ApJ, 554, 43

[4] R. Fender, S. Corbel, T. Tzioumis, V. McIntyre, D. Campbell-Wilson, et al., 1999, ApJ, 519, L165

[5] J. Homan, \& T. Belloni, 2005, [astro-ph/ 0412597 ]

[6] J. Homan, M. Buxton, S. Markoff, C. B. Bailyn, E. Nespoli \& T. Belloni, 2005, ApJ, 624, 295

[7] A. K. H. Kong, P. A. Charles, E. Kuulkers, \& S. Kitamoto, 2002, MNRAS, 329, 588

[8] J. E. McClintock, \& R. A. Remillard, 2004, [astro-ph / $0306213 v 4$ ]

[9] G. B. Rybicki \& A. P. Lightman, 1986, Radiative Transfer in Astrophysics, Wiley-VCH

[10] J. Rodriguez, S. Corbel, \& J. A. Tomsick, 2003, ApJ, 595, 1032

[11] J. Rodriguez, S. E. Shaw, D. C. Hannikainen, T. Belloni, S. Corbel, et al., 2007, ApJ, 675, 1449 